\title{
Stability Analysis of Nonlinear Quadratic Systems via Polyhedral Lyapunov Functions
}

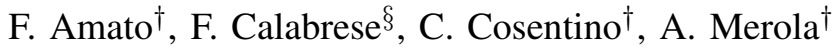

\begin{abstract}
Quadratic systems play an important role in the modeling of a wide class of nonlinear processes (electrical, robotic, biological, etc.). For such systems it is of mandatory importance not only to determine whether the origin of the state space is locally asymptotically stable, but also to ensure that the operative range is included into the convergence region of the equilibrium. Based on this observation, this paper considers the following problem: given the zero equilibrium point of a nonlinear quadratic system, assumed to be locally asymptotically stable, and a certain polytope in the state space containing the origin, determine whether this polytope belongs to the region of attraction of the equilibrium. The proposed approach is based on polyhedral Lyapunov functions, rather than on the classical quadratic Lyapunov functions. An example shows that our methodology may return less conservative results than those obtainable with previous approaches.
\end{abstract}

\section{INTRODUCTION}

Nonlinear quadratic systems provide an appropriate tool for modeling phenomena in a wide range of applications both in engineering (electric power systems [1], chemical reactors [2], and robots [3]), and in other areas such as biology, ecology, economics and meteorology [4].

For example, in the field of biology, the study of the behavior of quadratic systems is motivated by the fact that the interactions between different (biochemical and biological) species, e.g. in enzymatic reaction kinetics [5] or in Lotka-Volterra prey predator models [6], [7], are described by a quadratic law.

In such applications, it is relevant to determine the set of initial conditions around an equilibrium point which lead the systems trajectories to the equilibrium itself. This requires to obtain an estimate of the domain of attraction (DA) of the equilibrium.

The study of the DA of equilibrium points of nonlinear systems has a long history in the mathematical literature and an increasing interest in the field of systems theory. The main results, which are settled in the Lyapunov theory of stability, approximate the domain of attraction through a level curve of a given Lyapunov function.

In the current literature, quadratic Lyapunov functions, which allow to compute an ellipsoidal estimate of the DA of nonlinear (quadratic, cubic and polynomial) systems,

F. Amato, C. Cosentino and A. Merola are with the School of Computer Science and Biomedical Engineering, Università degli Studi Magna Græcia di Catanzaro, Via T. Campanella 115, 88100 Catanzaro, Italy.

${ }^{\S} \mathrm{F}$. Calabrese is with the Dipartimento di Informatica e Sistemistica, Università degli Studi di Napoli Federico II, Via Claudio 21, 80125 Napoli, Italy. are mainly used (e.g. [8], [9], [10], [11], [12]). Quadratic Lyapunov functions may provide conservative results, especially in the case of a priori choice of the function itself. Some optimization procedures are proposed in order to improve the estimate. In particular, in [9], a quadratic Lyapunov function is computed in order to maximize the volume of the estimated DA through the solution of a non-convex optimization problem. In [13], [10], a convex optimization procedure based on Linear Matrix Inequalities (LMIs) allows to compute a sub-optimal candidate for the quadratic Lyapunov function, by using a gradient search algorithm. This approach is hardly treatable from the numerical point of view, especially for the case of non-odd polynomial systems (such as quadratic systems).

Since the exact determination of the whole DA of the equilibrium point of a given quadratic system is a difficult or even impossible task (except for very simple cases), in the recent papers [14] and [15] an approach is proposed to solve the more practical problem of determining whether an assigned polytope $\mathcal{P}$ containing the origin of the state space belongs to the DA of the equilibrium. Polytopes, and more specifically boxes, naturally arise whenever the operative range around the equilibrium point is given in terms of admissible intervals of variation for each state variable.

In the same context of [14] and [15], it is expected that the use of polyhedral Lyapunov functions [16], [17], [18], [19] might lead to less conservative results. Indeed in [14], [15], the proposed approach requires to cover the given polytope $\mathcal{P}$ by an ellipse and then establish whether the ellipse belongs to the DA; this follows from the fact that the level curves of a quadratic function are ellipses. Conversely, the level curves of a polyhedral function are polytopes, and therefore they better fit the set $\mathcal{P}$. To this regard, a numerical example, provided at the end of the paper, illustrates the goodness of the proposed approach with respect to the previous literature.

The paper is organized as follows. In Section II the problem we deal with in the paper is precisely stated and some preliminary definitions and results about polytopes are provided. Also, a novel procedure to pass from the description of a polytope as convex hull of a finite set of points to the description of the same polytope through a full row rank matrix, is given. This procedure is used in Section III to derive the main result of the paper, namely a sufficient condition guaranteeing that a given polytope $\mathcal{P}$ belongs to the DA of a given quadratic system. In Section IV a numerical example shows the goodness of the proposed 
technique over existing methods. Finally some concluding remarks are given in section $\mathrm{V}$.

\section{Problem Statement and Preliminaries}

In this paper we consider quadratic systems, defined as

$$
\dot{x}=A x+B(x),
$$

where $x \in \mathbb{R}^{n}$ is the system state and

$$
B(x)=\left(\begin{array}{c}
x^{T} B_{1} x \\
x^{T} B_{2} x \\
\vdots \\
x^{T} B_{n} x
\end{array}\right)
$$

with $B_{i} \in \mathbb{R}^{n \times n}, i=1, \ldots, n$.

If $x_{e} \neq 0$ is an equilibrium point for system (1), then

$$
A x_{e}+B\left(x_{e}\right)=0 .
$$

From (3), by letting

$$
z=x-x_{e},
$$

the resulting system

$$
\begin{aligned}
\dot{z} & =\left(A+2\left(\begin{array}{c}
x_{e}^{T} B_{1} \\
x_{e}^{T} B_{2} \\
\vdots \\
x_{e}^{T} B_{n}
\end{array}\right)\right) z+B(z)+A x_{e}+B\left(x_{e}\right) \\
& =\left(A+2\left(\begin{array}{c}
x_{e}^{T} B_{1} \\
x_{e}^{T} B_{2} \\
\vdots \\
x_{e}^{T} B_{n}
\end{array}\right)\right) z+B(z)
\end{aligned}
$$

is a quadratic one in form (1).

Since we showed above that zero equilibrium point of the transformed system (5) corresponds to the equilibrium $x=x_{e}$ of system (1), without loss of generality, we focus on the stability properties of the zero equilibrium point of system (1). Moreover, through a slight abuse of terminology, with "stability properties" of system (1), it is meant "stability properties of the zero equilibrium point" of system (1).

Checking local asymptotic stability of system (1) is rather simple, since it amounts to evaluate the eigenvalues location of the linearized system $\dot{x}=A x$. In practical engineering applications, however, establishing the simple local asymptotic stability is not sufficient, since it is often required to obtain an estimate of the DA.

Our goal in this paper is to solve the following problem.

Problem 1: Assume that the matrix $A$ in (1) is Hurwitz; then, given a polytope $\mathcal{P} \subset \mathbb{R}^{n}$, with 0 an interior point of $\mathcal{P}$, establish whether $\mathcal{P}$ belongs to the DA of system (1). $\diamond$

Remark 1: In most of the engineering applications the operative range of a nonlinear system is assigned in terms of the variation range of the state variables; therefore the polytope $\mathcal{P}$ often reduces to a box. In any case polytopes can approximate at will any compact region in $\mathbb{R}^{n}$.
In [14], [15] a LMIs optimization procedure is proposed in order to solve Problem 1. Roughly speaking, such procedure tries to cover the polytope $\mathcal{P}$ by an ellipsoid whose boundary is the level curve of a quadratic Lyapunov function. This kind of approach is obviously inherently conservative because, depending on the shape of $\mathcal{P}$, there may be a lot of space between the covering ellipsoid and the set $\mathcal{P}$.

It is expected that an approach based on polyhedral Lyapunov function might lead to less conservative results, since the level curves of polyhedral functions are polytopes, which provide a more natural fitting of the set $\mathcal{P}$.

\section{A. Some useful results about polytopes}

In the following we provide some preliminary definitions and results on linear algebra and polytopes which will be useful to state the main result of the paper.

Definition 1 (Affine space): An affine space over a field $\mathbb{K}$ is a triplet $(A, V, \pi)$ composed of a nonempty set $A$, a vector space $V$ over $\mathbb{K}$ and an application $\pi:(a, b) \in$ $A \times A \rightarrow \pi(a, b) \in V$ such that

i) for all $a \in A$ and $v \in V$, there exists a unique point $b \in A$ such that $\pi(a, b)=v$;

ii) $\forall a, b, c \in A, \pi(a, b)+\pi(b, c)=\pi(a, c)$.

For example, $\mathbb{R}^{n}$ can be interpreted both as a points set and as a vector space. Indeed to a given point $a \in \mathbb{R}^{n}$ we can associate the vector $v_{a} \in \mathbb{R}^{n}$ going from the origin to the point $a$. It is simple to verify that the triplet $\left(\mathbb{R}^{n}, \mathbb{R}^{n}, \pi\right)$ is an affine space when

$$
\pi(a, b):=v_{a}-v_{b} .
$$

Definition 2 (Affine subspace): Let $(A, V, \pi)$ be an affine space. Let $H$ be a subset of $A$ and $V_{H}$ the set of vectors $\{\pi(a, b): a, b \in H\}$. Let us restrict the domain and codomain of $\pi$ to $H \times H$ and $V_{H}$, respectively, and denote the resulting application with $\pi_{H}$. The triplet $\left(H, V_{H}, \pi_{H}\right)$ is an affine subspace of $(A, V, \pi)$ if a) $V_{H}$ is a vector subspace of $V$ and b) $\left(H, V_{H}, \pi_{H}\right)$ is an affine space. The dimension of the affine subspace $\left(H, V_{H}, \pi_{H}\right)$ is the dimension of the vector subspace $V_{H}$.

Let us consider the affine space $\left(\mathbb{R}^{2}, \mathbb{R}^{2}, \pi\right)$, with $\pi$ defined as in (6), and the line $L:=\left\{x \in \mathbb{R}^{2}: x_{1}+x_{2}=1\right\} \subset \mathbb{R}^{2}$. Note that $R_{L}^{2}$ is the subspace of $R^{2}$ given by the bisector of the second and fourth quadrant. It is simple to recognize that the triplet $\left(L, \mathbb{R}_{L}^{2}, \pi_{L}\right)$ is an affine subspace of $\left(\mathbb{R}^{2}, \mathbb{R}^{2}, \pi\right)$ of dimension one.

In the following we will consider the affine space associated to the standard vector space $\mathbb{R}^{n}$ over the field $\mathbb{R}$, and the related affine subspaces; correspondingly, the operator $\pi(a, b)$, with $a, b \in \mathbb{R}^{n}$, will always coincide with the one defined in (6). Concerning Definition 2, without loss of generality and for the sake of simplicity, we shall refer to the "affine subspace $H$ " rather than to the "affine subspace $\left(H, V_{H}, \pi_{H}\right)$ ". 
Definition 3 (Convex and affine hull [20]): Given a set $A \subset \mathbb{R}^{n}$ the convex hull of $A$ is defined as the subset of $\mathbb{R}^{n}$ composed of all vectors obtained via convex combination from the elements of $A$, namely

$$
\begin{aligned}
\operatorname{conv}(A):= & \left\{v \in \mathbb{R}^{n}: v=\sum_{i=1}^{k} \lambda_{i} v^{(i)}, \sum_{i=1}^{k} \lambda_{i}=1,\right. \\
& \left.\lambda_{i} \geq 0, v^{(i)} \in A, i=1, \ldots, k, k=1,2, \ldots\right\} ;
\end{aligned}
$$

if in (7) we eliminate the requirement that the numbers $\lambda_{i}$ are nonnegative, the resulting set is said to be the affine hull of $A$.

It is worth noticing that the convex hull of a set $A$ is the smallest convex set containing $A$, while the affine hull turns out to be an affine subspace of $\mathbb{R}^{n}$.

If we deal with a finite set, say $K=\left\{x^{(1)}, \ldots, x^{(k)}\right\} \subset$ $\mathbb{R}^{n}$, the convex hull of $K$ turns out to be a polytope, whose dimension [20] is given by the dimension of the affine hull of $K$. Moreover, as stated in the next lemma, the set of vertices of a given polytope $\mathcal{P}$ is a subset of $K$.

Lemma 1 ([20]): Given a polytope defined as the convex hull of $K=\left\{x^{(1)}, \ldots, x^{(k)}\right\} \subset \mathbb{R}^{n}$, the vertices of the polytope are the points $x^{(i)} \in K$ which satisfy the following property

$$
x^{(i)} \notin \operatorname{conv}\left(K-\left\{x^{(i)}\right\}\right) .
$$

Remark 2: Note that, given a collection of symmetric points $K=\left\{x^{(1)}, \ldots, x^{(2 l)}\right\}, x^{(i)}=-x^{(l+i)}, i=1, \ldots, l$, if $x^{(i)}$ is a vertex of $\operatorname{conv}(K)$, then also $x^{(l+i)}=-x^{(i)}$ is a vertex of $\operatorname{conv}(K)$.

In this paper we shall focus on polytopes symmetrical with respect to the origin of $R^{n}$. To this regard note that, given any symmetrical polytope $\mathcal{P} \subset \mathbb{R}^{n}$, there always exists a full row rank matrix $Q \in \mathbb{R}^{n \times m}, m \geq n$, such that the polytope $\mathcal{P}$ can be alternatively defined as [21]

$$
\mathcal{P}=\wp(Q):=\left\{x \in \mathbb{R}^{n}:\left\|Q^{T} x\right\|_{\infty} \leq 1\right\},
$$

where, given a vector $v \in \mathbb{R}^{n},\|v\|_{\infty}:=\max _{i=1, \ldots, n}\left|v_{i}\right|$ is the infinity norm of $v$.

Therefore a given symmetric polytope $\mathcal{P}$ admits two different equivalent descriptions. As we shall see later, a fundamental point in our approach will be the development of an efficient algorithm to pass from the representation of $\mathcal{P}$ as convex hull of its vertices to the matrix description (8) of $\mathcal{P}$.

Definition 4 (Affinely independent points [20]): A set of $k>0$ points is affinely independent if its affine hull has dimension $(k-1)$.

Lemma 2 ([20]): The convex hull of any $(n+1)$ affinely independent points in $\mathbb{R}^{n}$ is a polytope of dimension $n$. $\diamond$

Finally, the next definition generalizes for our purposes, the concept of "points in general position" given in [20].

Definition 5 (Set of points in generic position): A set of $k \geq n$ points in $\mathbb{R}^{n}, n \geq 2$, is said to be in generic position if there is no $n$-tuple composed of such points which lies on a common affine plane of dimension $(n-2)$. If $n=1$ any set of points is in generic position.

Remark 3: Note that, requiring that $k$ points are in generic position in $\mathbb{R}^{n}$, implies that, $i$ ) they are distinct for $n=2$; ii) there is no triplet of such points which lies on a common line for $n=3$; iii) there is no quadruplet of such points which lies on a common plane for $n=4$; etc. $\diamond$

Remark 4: The minimum number of vertices that define a symmetric polytope in $\mathbb{R}^{n}$ of dimension $n$ is $2 n$. An example of symmetric polytope with minimum number of vertices is the crosspolytope of dimension $n$

$\mathcal{C}:=\left\{x \in \mathbb{R}^{n}: \sum_{i}\left|x_{i}\right| \leq 1\right\}=\operatorname{conv}\left\{e_{1},-e_{1}, \ldots, e_{n},-e_{n}\right\}$,

where $e_{i}$ are the unit vectors in $\mathbb{R}^{n}$. Note that these points are in generic position.

\section{B. Machinery}

The solution to the following Problem will be useful to derive the main result of the paper.

Problem 2: Given a polytope $\mathcal{P}$ defined as the convex hull of $2 l$ symmetric points $K=\left\{x_{Q}^{(1)}, \ldots, x_{Q}^{(2 l)}\right\}$ in generic position in $\mathbb{R}^{n}, x_{Q}^{(i)}=-x_{Q}^{(l+i)}, i=1, \ldots, l$, with $l \geq n$, where $(n+1)$ of them are affinely independent, find a matrix $Q$ such that (8) is satisfied.

The following procedure solves Problem 2.

Procedure 1 (Solution to Problem 2): First of all, note that Lemma 2 guarantees that $\mathcal{P}$ has dimension $n$. Next, using Lemma 1 , it is possible to select the $2 k$ vertices of $\mathcal{P}, k \leq l$, from $\left\{x_{Q}^{(1)}, \ldots, x_{Q}^{(2 l)}\right\}$. Let us reorder the points such that $\left\{x_{Q}^{(1)}, \ldots, x_{Q}^{(k)}, x_{Q}^{(l+1)}, \ldots, x_{Q}^{(l+k)}\right\}$ are the vertices of $\mathcal{P}$. Note that, given the assumption that the set of points is in generic position, each vertex $x_{Q}^{(i)}, i=$ $1, \ldots, k$, of $\mathcal{P}$ is the intersection of $s_{i}$ half-planes $q_{i, h} \in$ $\mathbb{R}^{n}, h=1, \ldots, s_{i}, s_{i} \geq n$; such half-planes are univocally determined by $n$ vertices. These half-planes are columns vectors which satisfy, for $i=1, \ldots, k$, the following conditions

a)

$$
q_{i, h}^{T} x_{Q}^{(i)}=1
$$

b) there exists a $(n-1)$-tuple of indexes $i_{1} \neq i_{2} \neq \cdots \neq$ $i_{n-1} \in\{1, \ldots, k, l+1, \ldots, l+k\}-\{i\}$ such that

$$
\begin{aligned}
q_{i, h}^{T} x_{Q}^{\left(i_{t}\right)}=1, \quad \forall t & =1, \ldots, n-1 \\
q_{i, h}^{T} x_{Q}^{(j)} \leq 1, \quad \forall j \in & \{1, \ldots, k, l+1, \ldots, l+k\}- \\
& -\left\{i, i_{1}, \ldots, i_{n-1}\right\} .
\end{aligned}
$$

Once the half-planes $q_{i, h}$ have been found, we can equivalently define the polytope $\mathcal{P}$ as in (8), where the matrix $Q$ can be constructed as follows

$$
Q=\left(\begin{array}{lllllll}
q_{1,1} & \ldots & q_{1, s_{1}} & \ldots & q_{k, 1} & \ldots & q_{k, s_{k}}
\end{array}\right) .
$$


Remark 5: It is easy to see that for all $i=1, \ldots, k$ and $h=1, \ldots, s_{i}$, there exist at least $(n-1)$ vertices $i_{1}, \ldots, i_{n-1}$ such that one of its associate half-planes is equal to $q_{i, h}$, i.e. $q_{i, h}=q_{i_{t}, m}, m_{t} \in\left\{1,2, \ldots, s_{i_{t}}\right\}$. Therefore the matrix $Q$ presents several repeated columns that, without loss of generality, can be canceled in order to lighten the computational burden.

In the sequel we will make use of the following definition.

Definition 6 (Candidate set of points): A collection of points $K=\left\{x_{Q}^{(1)}, \ldots, x_{Q}^{(2 l)}\right\} \subset \mathbb{R}^{n}$, with $l \geq n$, is said to be a candidate set of points if

- the points are in generic position in $\mathbb{R}^{n}$;

- the points are symmetric, i.e. $x_{Q}^{(i)}=-x_{Q}^{(l+i)}, i=$ $1, \ldots, l$;

- $(n+1)$ of the points are affinely independent.

Without any loss of generality, we assume that the vertices of the polytope defined as $\operatorname{conv}(K)$ are the first $k$ points, $k \leq l$, of $K$ and their symmetric. Finally, we denote by $q_{i, h}, h=1, \ldots, s_{i}$, the half-planes associated to the vertex $x_{Q}^{(i)}, i=1, \ldots, k$, of the polytope.

\section{MAIN RESULT}

In order to solve Problem 1, we make use of the class of polyhedral Lyapunov functions, which are piecewise linear functions of the following form

$$
V(x)=\left\|Q^{T} x\right\|_{\infty},
$$

where $Q \in \mathbb{R}^{n \times l}$ is a full row rank matrix.

Given a generic system in the form $\dot{x}=f(x)$ and a Lyapunov function $V(x)$, we recall the definition of Dini (upper) derivative [22] of $V(x)$ along the solutions of the system:

$$
\dot{V}(x)=\left.\lim \sup _{\tau \rightarrow 0^{+}} \frac{V(x+\tau \dot{x})-V(x)}{\tau}\right|_{\dot{x}=f(x)} .
$$

Such definition returns the classical derivative when $V(x)$ is continuously differentiable, but also enables to treat the more general case in which the Lyapunov function is not differentiable everywhere (as it is the case of polyhedral functions).

For a polyhedral function (12) associated to the system $\dot{x}=f(x)$, the derivative is

$$
\dot{V}(x)=\max _{j \in I(x)} \tilde{q}_{j}^{T} f(x),
$$

where $\tilde{Q}=\left(\begin{array}{ll}Q & -Q\end{array}\right), \tilde{q}_{j}$ denotes the $j$-th column of $\tilde{Q}$ and $I(x)$ is the set of the indexes $j$ such that $V(x)=\tilde{q}_{j}^{T} x$ (see [19]).

We recall the following theorem.

Theorem 1 ([23]): A given closed set $\mathcal{E} \subset \mathbb{R}^{n}$, with 0 an interior point of $\mathcal{E}$, is an estimate of the DA of system (1) if

i) $\mathcal{E}$ is an invariant set for system (1);

ii) there exists a Lyapunov function $V(x)$ such that

a) $V(x)$ is positive definite on $\mathcal{E}$; b) $\dot{V}(x)$ is negative definite on $\mathcal{E}$.

Now we are ready to state the main result of the paper, which is a sufficient condition to guarantee a positive answer to the question posed by Problem 1. Essentially, the proof is based on the construction of a polyhedral Lyapunov function which satisfies the hypotheses of Theorem 1, where $\mathcal{E}$ turns out to be a polytope whose boundary is a level curve of the polyhedral function itself. This guarantees that $\mathcal{E}$ is an invariant set. Note that a key point in the proof is the assumption that the system under consideration is quadratic; therefore the technique cannot be extended to higher order systems (cubic or, more generally, polynomial).

Theorem 2: The polytope $\mathcal{P}$, with $0 \in \mathcal{P}$, belongs to the DA of system (1) if there exist a candidate set of points in the sense of Definition 6 such that

$$
\operatorname{conv}(K) \supseteq \mathcal{P}
$$

and the following condition holds for all $i=1, \ldots, k, h=$ $1, \ldots, s_{i}, j=1, \ldots, k, l+1, \ldots, l+k$,

$$
q_{i, h}^{T}\left[A+\left(\begin{array}{c}
x_{Q}^{(j) T} B_{1} \\
x_{Q}^{(j) T} B_{2} \\
\vdots \\
x_{Q}^{(j) T} B_{n}
\end{array}\right)\right] x_{Q}^{(i)}<0 .
$$

Proof: Let $K=\left\{x_{Q}^{(1)}, \ldots, x_{Q}^{(2 l)}\right\}$ such that $\mathcal{E}:=$ $\operatorname{conv}(K) \supseteq \mathcal{P}$. By using Procedure 1 , determine the halfplanes $q_{i, h}$ associated to the $2 k$ vertices, $k \leq l$, and construct the $Q$ matrix as

$$
Q=\left(\begin{array}{lllllll}
q_{1,1} & \ldots & q_{1, s_{1}} & \ldots & q_{k, 1} & \ldots & q_{k, s_{k}}
\end{array}\right),
$$

such that $\mathcal{E}=\wp(Q)$.

Let us consider the candidate polyhedral Lyapunov function

$$
V(x)=\left\|Q^{T} x\right\|_{\infty}
$$

We denote by $\dot{V}$ the Dini derivative of $V$ along the solutions of system (1); in view of (13) we obtain

$$
\dot{V}(x)=\max _{j \in I(x)} \tilde{q}_{j}^{T}\left[A+\left(\begin{array}{c}
x^{T} B_{1} \\
x^{T} B_{2} \\
\vdots \\
x^{T} B_{n}
\end{array}\right)\right] x,
$$

where $\tilde{Q}=\left(\begin{array}{ll}Q & -Q\end{array}\right)$ and $I(x)$ is the set of the indexes $j$ such that $V(x)=\tilde{q}_{j}^{T} x$.

For $p \in \mathcal{E}$, let us define

$$
A_{M}(p):=A+\left(\begin{array}{c}
p^{T} B_{1} \\
p^{T} B_{2} \\
\vdots \\
p^{T} B_{n}
\end{array}\right)
$$

note that $A_{M}(p)$ is an affine function of $p$. 
We have, for all $x \in \mathcal{E}$,

$$
\begin{aligned}
\dot{V}(x) & \leq \max _{p \in \mathcal{E}} \max _{j \in I(x)} \tilde{q}_{j}^{T} A_{M}(p) x \\
& =\max _{j \in I(x)} \max _{p \in \mathcal{E}} \tilde{q}_{j}^{T} A_{M}(p) x \\
& =\max _{j \in I(x)} \max _{p \in \operatorname{vert}(\mathcal{E})} \tilde{q}_{j}^{T} A_{M}(p) x \\
& =\max _{p \in \operatorname{vert}(\mathcal{E})} \max _{j \in I(x)} \tilde{q}_{j}^{T} A_{M}(p) x,
\end{aligned}
$$

where $\operatorname{vert}(\mathcal{E})$ denotes the set of the vertices of $\mathcal{E}$ and we have used the fact that $\tilde{q}_{j}^{T} A_{M}(p) x$ is an affine function of $p$.

Now, it is simple to recognize that, for a given $p$, the sign behavior for $x \in \mathcal{E}$ of the function $\max _{j \in I(x)} \tilde{q}_{j}^{T} A_{M}(p) x$ can be inferred by the sign behavior on the boundary of the polytope $\mathcal{E}$; moreover the maximum value of the linear function

$$
\tilde{q}_{j}^{T} A_{M}(p) x
$$

on the $j$-th face of $\mathcal{E}$ is attained at the vertices of the face itself. Hence, from (18) we have that $\dot{V}(x)$ is negative definite for all $x \in \mathcal{E}$ if

$$
\max _{p \in \operatorname{vert} \mathcal{E}} \max _{j \in I\left(x_{Q}^{(i)}\right)} \tilde{q}_{j}^{T} A_{M}(p) x_{Q}^{(i)}<0,
$$

for all $i=1, \ldots, k, l+1, \ldots, l+k$. The symmetry of the polytope implies that (19) is equivalent to

$$
\max _{p \in \operatorname{vert}(\mathcal{E})} \max _{j \in I\left(x_{Q}^{(i)}\right)} q_{j}^{T} A_{M}(p) x_{Q}^{(i)}<0,
$$

for all $i=1, \ldots, k$.

Finally, for a given $i$, the set $\left\{q_{j}, j \in I\left(x_{Q}^{(i)}\right)\right\}$ is equal to the set $\left\{q_{i, h}, h=1, \ldots, s_{i}\right\}$; therefore we can conclude that $\dot{V}(x)$ is negative definite if

$$
\max _{p \in \operatorname{vert}(\mathcal{E})} q_{i, h}^{T} A_{M}(p) x_{Q}^{(i)}<0,
$$

for all $i=1, \ldots, k$, and $h=1, \ldots, s_{i}$, which is equivalent to $(15)$.

Therefore the hypotheses of the theorem guarantee the existence of a positive definite polyhedral Lyapunov function $V(x)=\left\|Q^{T} x\right\|_{\infty}$ with a negative definite derivative on $\mathcal{E}$. This, together with the fact that the polytope $\mathcal{E}$ is an invariant set (the boundary of $\mathcal{E}$ is a level curve of the Lyapunov function $V(x)$ ), allows to apply Theorem 1 which guarantees that $\mathcal{E}$ is an estimate of the DA. The proof follows from the inclusion (14), since $\mathcal{E}=\operatorname{conv}(K)$.

Remark 6: A possible choice of the candidate set of points in Theorem 2 is $\operatorname{vert}(\mathcal{P})$ since (14) is clearly verified.

In order to find a candidate set of points satisfying the conditions of Theorem 2, the following procedure can be used.

Procedure 2 (Implementation of Theorem 2): The procedure we propose to find a candidate set of points satisfying the conditions of Theorem 2 is the following.

1) Fix a number $l \geq n$. Let $K_{0}=\left\{x_{Q}^{(i)}\right\}_{i=1, \ldots, 2 l}$ be a candidate set of points, in the sense of Definition 6 , whose convex hull is a regular polytope of $2 l$ vertices which contains $\mathcal{P}$.

2) Find a candidate set of points $K$ solving the problem

$$
\min _{K} \max _{\substack{j=1, \ldots, k \\ l+1, \ldots, l+k}} f_{j}(K)
$$

with initial condition $K_{0}$, where

$$
f_{j}(K)=\max _{i=1, \ldots, k, k=1, \ldots, s_{i}} q_{i, h}^{T}\left[A+\left(\begin{array}{c}
x^{(j) T} B_{1} \\
x^{(j) T} B_{2} \\
\vdots \\
x^{(j) T} B_{n}
\end{array}\right)\right] x_{Q}^{(i)} .
$$

3) Let $M=\min _{K} \max _{j} f_{j}(K)$. If $M<0$ then

$$
K_{\text {opt }}=\arg M,
$$

and go to 4), else set

$$
\begin{aligned}
K_{0} & =K \cup\left\{x_{Q}^{(l+1)},-x_{Q}^{(l+1)}\right\}, x_{Q}^{(l+1)} \in \mathbb{R}^{n} \\
l & =l+1
\end{aligned}
$$

and go to 2).

4) $K_{\text {opt }}$ is the candidate set of points satisfying the conditions of Theorem 2 .

Remark 7: To solve problem (21), we have made use of the Matlab Optimization Toolbox routine fminimax [24], with variables $x_{Q}^{(i)}, i=1, \ldots, l$.

Remark 8: The choice of $x_{Q}^{(l+1)}$ in step 3) is done putting such point on one of the faces of $\wp(Q)$. In particular, if $\max _{j} f_{j}(K)$ is obtained in correspondence of the vertex $x_{Q}^{(t)}$ and the vertex $j^{\star} \in \operatorname{vert}(\mathcal{P})$, the point is put in the middle of the face defined by the half-plane $q_{t, h}$ where

$$
q_{t, h}^{T}\left[A+\left(\begin{array}{c}
x^{\left(j^{\star}\right) T} B_{1} \\
x^{\left(j^{\star}\right) T} B_{2} \\
\vdots \\
x^{\left(j^{\star}\right) T} B_{n}
\end{array}\right)\right] x_{Q}^{(t)}
$$

reaches the maximum value. In this way, since at each step the algorithm begins from the solution found in the previous step, the value $M$ decreases (or, at least, does not increase) at each step.

\section{EXAMPLE}

In order the compare the results proposed in this paper with the previous literature on the same topic, we consider the numerical example proposed in [14]

$$
\left\{\begin{array}{l}
\dot{x}_{1}=-50 x_{1}-16 x_{2}+13.8 x_{1} x_{2} \\
\dot{x}_{2}=13 x_{1}-9 x_{2}+5.5 x_{1} x_{2} .
\end{array}\right.
$$

The polytope $\mathcal{P}$ is the box defined as follows

$$
\mathcal{P}:=[-1.2,1.2] \times[-2.8,2.8] .
$$

Note that the box $\mathcal{P}$ is larger than the box considered in [14]; moreover, the approach of [14] does not allow to establish whether $\mathcal{P}$ belongs to the DA of system (25) (the 


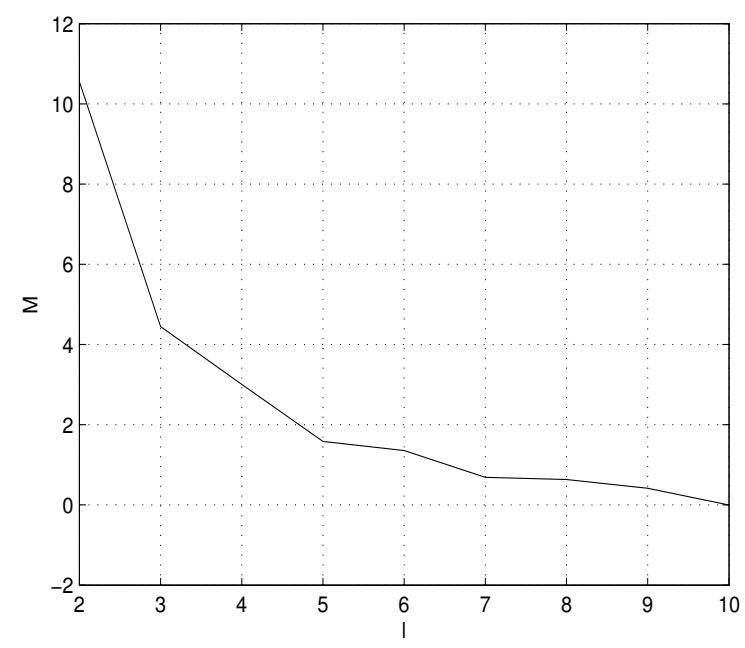

Fig. 1. Trend of the parameter $M$ in Procedure 2 when applied to the example.

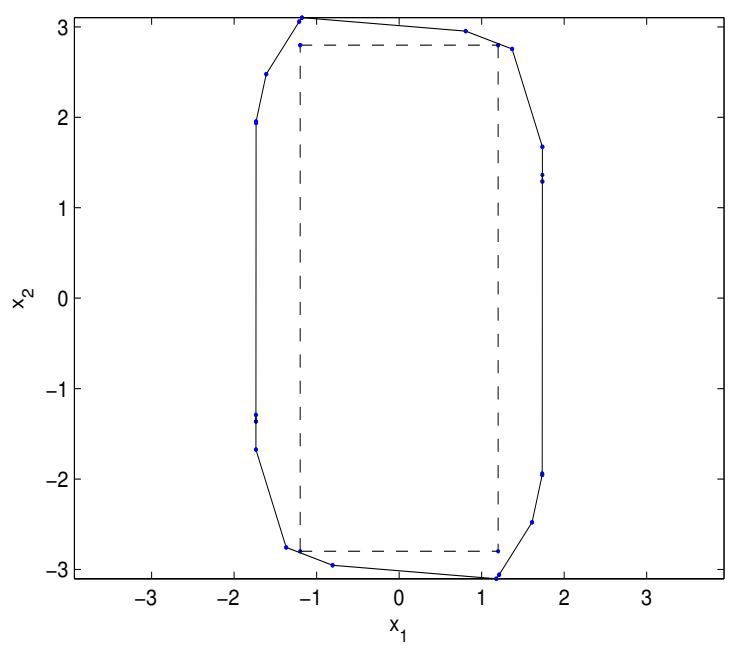

Fig. 2. Polyhedral Lyapunov function for the example. The box $\mathcal{P}$ (dashed line) and the level curve of the polyhedral Lyapunov function found by the procedure (solid line).

problem stated in [14] is found unfeasible when tested using the Matlab LMI Toolbox).

Conversely, by using the approach proposed in this paper, we are able to conclude that $\mathcal{P}$ belongs to the DA of system (25).

We started Procedure 2 with $l=2$ and the candidate set of points $\operatorname{vert}(\mathcal{P})$. Fig. 1 shows the trend of $M$ each time step 3) of Procedure 2 is reached, for different values of $l$. Fig. 2 shows the last polytope $\wp(Q)$ of 20 vertices $(l=10)$ whose vertices satisfy the conditions of Theorem 2 .

\section{CONCLUSION}

In this paper we have proposed a novel method to investigate the region of attraction of equilibrium points of quadratic systems. Given an asymptotically stable equilibrium point, the problem tackled in the present work is to ascertain whether a certain polytope, representing the admissible variations from the equilibrium, belongs to the DA. The proposed approach is based on the use of polyhedral Lyapunov functions, rather than on the classical quadratic Lyapunov functions. An example shows that our method may improve the results available in the literature.

\section{REFERENCES}

[1] T. Jyotika, V. Vittal, K. Wolgang, and A. Fouad, "Application of the normal form of vector fields to predict inter-area separation in power systems," IEEE Trans. Power Systems, vol. 12, pp. 844-580, 1997.

[2] O. E. Rössler, "Chaotic behavior in simple reaction system," Zeitschrift für Naturforsch A, vol. 31, pp. 259-264, 1976.

[3] L. Sciavicco and B. Siciliano, Modelling and Control of Robot Manipulators. McGraw Hill, 1996.

[4] F. Lorenz, "Deterministic non-periodic flow," Journal of Atmospheric Science, vol. 20, pp. 130-141, 1963.

[5] J. Murray, Mathematical Biology. Springer, New York, 2002.

[6] A. J. Lotka, Elements of physical biology. Williams \& Wilkins Co., Baltimore, 1925.

[7] V. Volterra, "Variazioni e fluttuazioni del numero d'individui in specie animali conviventi," Mem. R. Accad. Naz. dei Lincei Ser. VI, vol. 2, 1926.

[8] R. Genesio and A. Vicino, "Some results on the asymptotic stability of second-order nonlinear systems," IEEE Transaction on Automatic Control, vol. 29, pp. 857-861, 1984.

[9] A. N. Michel, N. R. Sarabudla, and R. K. Miller, "Stability analysis of complex dynamical systems Some computational methods," Circ. Syst. Signal Processing, vol. 1, pp. 561-573, 1982.

[10] G. Chesi, A. Garulli, A. Tesi, and A. Vicino, "Lmi-based computation of optimal quadratic lyapunov functions for odd polynomial systems," Int. Journal of Nonlinear and Robust Control, vol. 15, pp. 35-49, 2005.

[11] A. Tesi, F. Villoresi, and R. Genesio, "On the stability domain estimation via a quadratic Lyapunov function: convexity and optimality properties for polynomial systems," IEEE Transactions on Automatic Control, vol. 41, pp. 1650-1657, 1996.

[12] B. Tibken, "Estimation of the domain of attraction for polynomials systems via LMI's," in Proc. of the 39th IEEE International Conference on Decision and Control, Sidney, Australia, 2000.

[13] G. Chesi, A. Tesi, and A. Vicino, "Computing optimal quadratic Lyapunov function for polynomial nonlinear systems via LMIs," in Proc. of the 15th IFAC World Congress, Barcelona, 2002.

[14] F. Amato, C. Cosentino, and A. Merola, "On the Region of Asymptotic Stability of Nonlinear Quadratic Systems," in Proc. of the IEEE Mediterranean Symposium in Control and Automation, Ancona, Italy, 2006.

[15] — , "On the region of attraction of nonlinear quadratic systems," Automatica, vol. 43, pp. 2119-2123, 2007.

[16] R. K. Brayton and C. H. Tong, "Constructive stability and asymptotic stability of dynamical systems," IEEE Trans. on Circ. and Syst., vol. 27 , no. 11, pp. 1121-1130, 1980.

[17] —-, "Stability of dynamical systems: A constructive approach," IEEE Trans. on Aut. Contr., vol. 26, pp. 224-234, 1979.

[18] A. P. Molchanov and E. S. Pyatnitskii, "Lyapunov functions specifying necessary and sufficient conditions of absolute stability of nonlinear nonstationary control system," Autom. and Rem. Contr., vol. $47,1986$.

[19] F. Blanchini, "Nonquadratic Lyapunov functions for robust control," Automatica, vol. 31, no. 3, pp. 451-461, March 1995.

[20] G. M. Ziegler, Lectures on Polytopes. NY: Springer, 1998.

[21] R. T. Rockafellar, Convex Analysis. Princeton, NJ: Princeton University Press, 1972.

[22] W. Hahn, Stability of Motion. Berlin: Springer Verlag, 1967.

[23] H. K. Khalil, Nonlinear Systems. MacMillan, 1992.

[24] Optimization Toolbox 3, User's Guide. Natick, MA: The Mathworks, Inc., 2007. 